\title{
Relación entre el índice urémico y la función renal en pacientes con enfermedad renal crónica y en personas sanas
}

\author{
Relationship between uremic index and renal function in patients with chronic renal failure and in those \\ with normal renal function
}

Luis Enrique Cruz Llanos ${ }^{1, b}$; 2 , Javier Antonio Cieza Zevallos $1, \mathrm{a} ; 2, \mathrm{c}$

\section{RESUMEN}

Objetivo: Evaluar la relación entre el índice urémico (IU) y la función renal medida por el aclaramiento de creatinina $(\mathrm{ClCr})$ en personas sanas y en pacientes con enfermedad renal crónica (ERC); asimismo, comparar con la función renal extrapolada según la creatinina sérica y la calculada por algunas fórmulas recomendadas en la literatura internacional. Material y métodos: Estudio transversal, de correlación, analítico y observacional. Se incluyeron pacientes atendidos de forma ambulatoria en el Hospital Cayetano Heredia, Lima, Perú entre junio del 2018 y junio del 2019. Se realizó correlación de Pearson entre el IU y el ClCr; para la comparación de las medias del IU, creatinina sérica y las fórmulas estandarizadas en función al ClCr estratificado se utilizó el test de ANOVA y el eta cuadrado. Resultados: El IU de la población fue 4,37 $\pm 4,99 \mathrm{mg} / \mathrm{dl}$ y presentó correlación lineal cuadrática estadísticamente significativa con el $\mathrm{ClCr}(\mathrm{r}=-0,74, \mathrm{p}=0,000)$. Asimismo, el IU y el Log IU mostraron un valor predictivo del $\mathrm{ClCr}$ superior a la creatinina sérica y a las diversas fórmulas recomendadas en la literatura. El IU presentó correlación significativa con el potasio sérico y su fracción excretoria de forma relevante en comparación con la creatinina sérica. Conclusiones: El IU es un indicador del estado metabólico y nutricional que refleja el ClCr con una precisión estadísticamente significativa en la persona sana y en el paciente con distintos grados de ERC. Además, traduce aspectos relevantes de la función tubular renal.

PALABRAS CLAVE: Uremia, indicadores y reactivos, pruebas de función renal, insuficiencia renal crónica. (Fuente: DeCS BIREME).

\section{SUMMARY}

Objective: To evaluate the relationship between uremic index (UI) and renal function measured by creatinine clearance $(\mathrm{CrCl})$ in persons with normal renal function and in those with chronic renal failure (CRF), as well as to compare the renal function extrapolated from serum creatinine and that calculated with equations internationally recommended. Methods: A cross sectional study was conducted including patients attended in outpatient clinics

\footnotetext{
Servicio de Nefrología, Hospital Cayetano Heredia. Lima, Perú.

Facultad de Medicina Alberto Hurtado, Universidad Peruana Cayetano Heredia. Lima, Perú.

Médico Especialista en Nefrología;

Médico Residente de Nefrología;

Profesor Emérito
} 
at Hospital Cayetano Heredia in Lima from June 2018 to June 2019. Pearson's correlation between UI and $\mathrm{CrCl}$ was calculated; comparison of means of UI, serum creatinine and standard equations was done by ANOVA and eta square. Results: the overall UI was $4.37 \pm 4.99 \mathrm{mg} / \mathrm{dl}$ and correlated linearly with $\mathrm{CrCl}(\mathrm{r}=-0.74, \mathrm{p}=0.000)$. The $\mathrm{UI}$ and the $\log \mathrm{UI}$ were significantly more predictive of $\mathrm{CrCl}$ than serum creatinine and the international equations used. The UI correlated significantly with serum potassium than serum creatinine. Conclusions: The UI is a good indicator of the metabolic and nutritional status in persons with normal renal function and with CRF and reflects more accurately the $\mathrm{CrCl}$.

KEYWORDS: Uremia, indicators and reagents, kidney function tests, chronic renal insufficiency. (Source: MeSH NLM).

\section{INTRODUCCIÓN}

El ser humano saludable desde la adultez hasta la senectud experimenta un proceso dinámico en su estructura corporal y su metabolismo, que es resultado de la interacción entre el ambiente externo (aparato inmunológico) y el medio interno. La regulación de la homeostasis corporal es la función renal por excelencia y su valoración ha sido materia de investigación a lo largo de la historia ${ }^{(1)}$.

El primer biomarcador utilizado para medir la función renal fue la urea, considerada como la mayor fuente circulante de compuestos nitrogenados y un indicador valioso del catabolismo tisular en la persona sana, cuya producción hepática depende en parte del amoniaco proveniente del amonio generado a nivel renal, interacción que es relevante ya que tanto la urea sérica como la tasa de excreción de amonio regulan las funciones renales de concentración urinaria y de equilibrio ácido base respectivamente. Sin embargo, la urea presenta limitaciones en su interpretación pues depende de dos variables en forma directa: la ingesta proteica y el estado catabólico de la persona ${ }^{(2,3)}$.

Los pacientes en estadios iniciales de enfermedad renal crónica (ERC) presentan aumento discreto y sostenido de urea sérica como resultado del aumento del $50 \%$ de la reabsorción tubular de la urea, situación dependiente de la reducción del flujo tubular renal. Sin embargo, el paciente experimenta progresivamente anorexia y baja ingesta proteica como factor de autocontrol, que se traduce en niveles séricos de urea por debajo de lo esperado para el grado de disfunción renal ${ }^{(4,5)}$. Por esta razón, la valoración de la función renal a partir del aclaramiento de urea en orina de 24 horas (ClUr) estaría subestimada en un $50 \%-60 \%{ }^{\left({ }^{(}\right)}$.

Otro indicador del estado metabólico de la persona sana es la creatinina sérica, producto final del metabolismo de la creatina, un ácido orgánico nitrogenado que una vez fosforilado constituye una reserva rápidamente movilizable de fosfatos de alta energía en el músculo esquelético. Dada las características de producción endógena constante, bajo peso molecular y fácil disponibilidad, este biomarcador permite determinar con bastante exactitud la función renal a través de la medición directa del aclaramiento de creatinina $(\mathrm{ClCr})$. Sin embargo, el $\mathrm{ClCr}$ puede sobrestimar la función renal entre 10 a $50 \%$ porque la creatinina se secreta a nivel tubular proximal, que en las personas sanas puede ser aproximadamente 15\% $(4,6,7)$. En pacientes con ERC, la secreción tubular de creatinina puede aumentar hasta $50 \%$, razón por la cual se comporta como marcador tardío de disfunción renal, evidenciándose un incremento leve de los niveles séricos sólo cuando la función de filtración glomerular se ha reducido en $50-75 \%{ }^{(4,8)}$. A partir de ese punto, el incremento de creatinina sérica sigue un aumento exponencial. Sin embargo, al existir disminución de masa magra propia del paciente con ERC producto de la anorexia, se puede evidenciar niveles de creatinina por debajo de lo esperado e incluso "aparentemente normales".

Diversos estudios han mostrado el rol predictor de "muerte renal" de estos biomarcadores de la función renal en contexto de factores de riesgo sobre todo cardiovascular, documentándose que tanto valores altos como muy bajos se correlacionan con desenlaces fatales. La relación de la urea y la creatinina ha sido estudiada en diversas poblaciones, en las que se ha demostrado que valores altos se asocian a mayor mortalidad a corto y largo plazo ${ }^{(9,10,11,12)}$.

El uso combinado del ClUr y $\mathrm{ClCr}$ fue demostrado por Lubowitz y col. ${ }^{(13)}$, quienes evidenciaron que en pacientes con ERC, el aclaramiento de inulina por debajo de los $20 \mathrm{ml} / \mathrm{min}$ estaba mucho más estrechamente relacionado al aclaramiento medio 
de la urea y creatinina, herramienta de uso relevante en contexto de personas con función de filtración disminuidas y pobre masa muscular que podrían mostrar creatinina normal o solo discretamente disminuidas, pero con niveles séricos de urea elevadas, o en personas con niveles séricos de urea bajos, por hipocatabolismo transitorio, con creatinina sérica anormalmente elevadas.

En un paciente estable los niveles séricos de urea y creatinina reflejarían probablemente mejor la tasa de filtración glomerular dado que el flujo urinario estaría corregido y la excreción urinaria y la creatinina sérica serían las variables que se corregirían entre sí. De este modo, la relación de la urea y creatinina séricas podrían reflejar de forma asertiva la función renal.

En la literatura nacional se reporta índices de desnutrición elevados consecuencia del bajo consumo dietético de proteínas ${ }^{(14)}$, por lo que el uso de fórmulas construidas en base a otras realidades no reflejaría de forma precisa la función renal en nuestra población.

El estudio tuvo como objetivo evaluar la relación del IU con la función renal medida como $\mathrm{ClCr}$, en personas sanas y en pacientes con enfermedad renal crónica; asimismo, comparar con la función renal extrapolada según la creatinina sérica y la calculada por algunas fórmulas recomendadas en la literatura internacional.

\section{MATERIAL Y MÉTODOS}

Estudio transversal de correlación, analítico y observacional. La población estuvo constituida por personas sanas y con diferentes estadios de ERC mayores de 18 años de edad, con índice de masa corporal (IMC) entre 18 y $35 \mathrm{~kg} / \mathrm{m}^{2}$, que fueron atendidos de forma ambulatoria en el Hospital Cayetano Heredia, de Lima, Perú entre junio del 2018 y junio del 2019. Se calculó un tamaño de muestra mínimo de 74 sujetos para una correlación lineal simple aceptando una desviación estándar de 0,16 para la variable independiente; 0,16 para la probable correlación y un valor de lambda de 0,33 .

La medición sérica de urea y creatinina se hizo considerando un ayuno mínimo de ocho horas, la recolección de orina de 24 horas se realizó con estrictas medidas de higiene y siguiendo el protocolo: descarte de la primera orina de la mañana y a partir de ese momento se recolectó la orina en un recipiente durante 24 horas hasta la primera orina del día siguiente. para la medición de la urea y creatinina séricas y urinarias se utilizó el test enzimático basado en la reacción ureasa - glutamato deshidrogenasa y el test cinético y colorimétrico basado en la reacción de Jaffé, respectivamente.

Los datos de la población: edad, sexo, agua corporal total, IMC, IU, $\mathrm{ClCr}$, electrolitos séricos y urinarios fueron colectadas en un registro estructurado en formato electrónico.

El IU fue considerado variable independiente, cuantitativa continua, con escala de medición de intervalo. Se calculó según la fórmula:

$$
\mathrm{IU}=\left[\begin{array}{c}
\text { Urea sérica }(\mathrm{mg} / \mathrm{dl}) / 20] \\
(\mathrm{mg} / \mathrm{dl})
\end{array}\right.
$$

La función renal, expresada como el $\mathrm{ClCr}$, se consideró variable dependiente, cuantitativa continua, con escala de medición de intervalo, y fue corregida para $1,73 \mathrm{~m} 2$ de superficie corporal. Azoemia se definió como valor de urea sérica $\geq 40 \mathrm{mg} / \mathrm{dl} \mathrm{o}$ creatinina sérica $\geq 1,1 \mathrm{mg} / \mathrm{dl}$.

Los sujetos de estudio fueron considerados como: a) persona sana: eutrófica (IMC: $18-24 \mathrm{~kg}$ / $\mathrm{m}^{2}$ ), funcional (índice de Karnofsky $>70$ ), sin conocimiento de cualquier enfermedad crónica; b) persona enferma sin azoemia: persona con evidencia de cualquier comorbilidad crónica o enfermedad renal crónica funcional o estructural detectada y creatinina $<1,2 \mathrm{mg} / \mathrm{dl}$, sobrepeso (IMC: $24-29 \mathrm{~kg} / \mathrm{m}^{2}$ ) u obesidad $\left(\mathrm{IMC} \geq 30 \mathrm{~kg} / \mathrm{m}^{2}\right)$ y con Karnofsky $<70$; c) persona enferma con azoemia: persona con evidencia de cualquier comorbilidad crónica y enfermedad renal crónica funcional o estructural detectada y creatinina $>1,1 \mathrm{mg} / \mathrm{dl}$; y d) persona con enfermedad renal crónica definida y creatinina $>2 \mathrm{mg} / \mathrm{dl}$ o urea $>100 \mathrm{mg} / \mathrm{dl}$ y $\sin$ enfermedad aguda intercurrente.

Los datos se expresaron utilizando estadística descriptiva, considerando medias y desviación estándar (x \pm DS) para las variables continuas. Se realizó correlación de Pearson entre el índice de uremia y el $\mathrm{ClCr}$, tomando en cuenta las siguientes covariables de control: IMC, edad, creatinina urinaria. También se realizó comparación de medias en función del $\mathrm{ClCr}$ estratificado según los estadios reconocidos de función renal en relación al IU, la creatinina sérica, urea sérica y valores que sugieren el aclaramiento de creatinina resultantes de la fórmula peruana de Vásquez, y las recomendadas internacionalmente de Cockroft y 
CKD-EPI mediante el test de ANOVA para saber su significancia clínica y el eta cuadrado para apreciar su fuerza predictiva. Para el análisis estadístico se utilizó el paquete SPSS V18, aceptándose como significancia estadística un $\mathrm{p}<0,05$.

El estudio fue revisado y aprobado por el Comité de Ética del Hospital Cayetano Heredia.

\section{RESULTADOS}

Se incluyeron en el estudio 146 pacientes. La edad promedio fue 49,38 $\pm 19,17$ años; el $39 \%$ estuvo en la categoría sanos; $34,9 \%$ fueron enfermos sin retención nitrogenada, $13 \%$ enfermos con enfermedad crónica

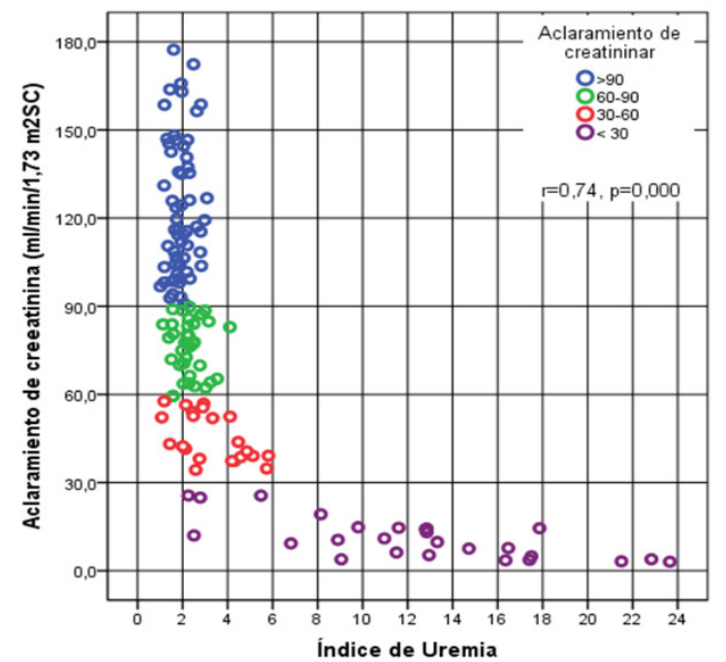

definida, pero con creatinina $<1,2 \mathrm{mg} / \mathrm{dl}$ o urea $<100$ $\mathrm{mg} / \mathrm{dl}$ y $13 \%$ enfermos con enfermedad renal crónica definida y creatinina $>2 \mathrm{mg} / \mathrm{dl} /$ o urea $>100 \mathrm{mg} / \mathrm{dl}$. El $53,7 \%$ fue de sexo femenino. $\mathrm{El} \mathrm{ClCr}$ de toda la población fue $70,6 \pm 39,3 \mathrm{ml} / \mathrm{min} / 1,73 \mathrm{~m}^{2}$; el valor promedio de creatinina sérica $1,9 \pm 2,6 \mathrm{mg} / \mathrm{dl}$ y el de urea sérica 49,4 $\pm 50,3 \mathrm{mg} / \mathrm{dl}$. El IMC promedio fue $25,75 \pm 5,02 \mathrm{~kg} / \mathrm{m}^{2}$; el $58,5 \%$ de los pacientes fueron eutróficos, $24 \%$ presentó sobrepeso y $17,5 \%$ tuvo obesidad.

El IU de la población total fue $4,37 \pm 4,99 \mathrm{mg} / \mathrm{dl}$ y tuvo correlación lineal cuadrática estadísticamente significativa con el aclaramiento de creatinina $(\mathrm{r}=$ $0,74, \mathrm{p}=0,000)$, la creatinina sérica $(\mathrm{r}=-0,72 \mathrm{p}=0,000)$

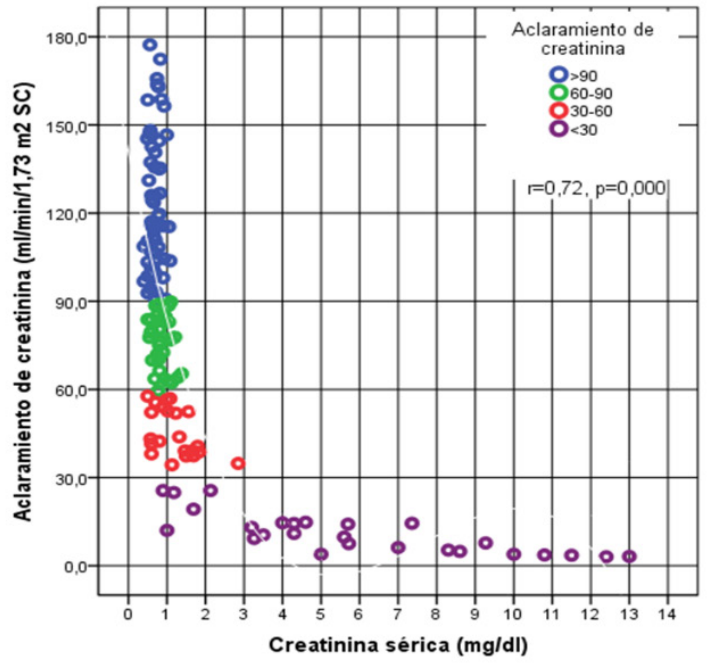

Gráfico 1. Relación del índice de uremia y la creatinina sérica con el aclaramiento de creatinina.

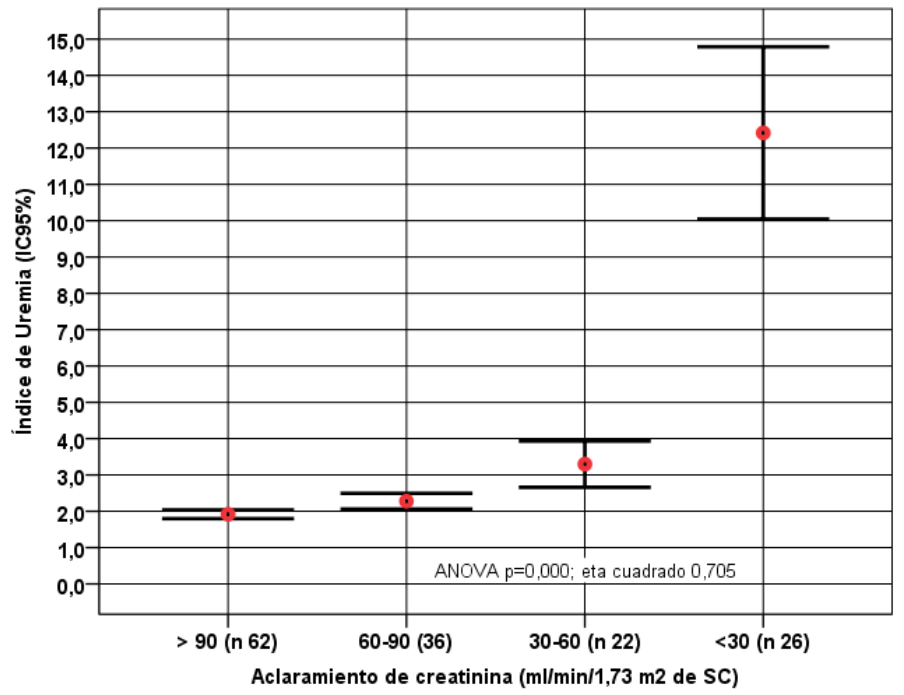

Gráfico 2. Índice de uremia y aclaramiento de creatinina según estadios de función renal. 
y la urea sérica $(\mathrm{r}=0,71, \mathrm{p}=0,000)$ (gráfico 1$)$. El gráfico 2 muestra la asociación entre el $\mathrm{IU}$ y el $\mathrm{ClCr}$ estratificado según el estadio de función renal.

Al analizar la fuerza de asociación entre el IU, la creatinina sérica y la urea sérica con el $\mathrm{ClCr}$ estratificado (mayor de 90, entre 60 y 90, entre 30 y 60 y menor de $30 \mathrm{ml} / \mathrm{min} / 1,73 \mathrm{~m}^{2} \mathrm{SC}$ ) se encontró asociación estadística entre las tres variables, con una fuerza de asociación discretamente diferenciada (tabla $1)$.

$\mathrm{Al}$ analizar si la correlación entre las dos variables mejoraba al ser corregido con el logaritmo del IU, se encontró que la correlación lineal fue $\mathrm{r}=0,728, \mathrm{p}=0,001$. Sin la corrección logarítmica la correlación fue 0,669. El gráfico 3 muestra la asociación del logaritmo del IU con el ClCr estratificado según estadios de función renal.

Tabla 1. Creatinina y urea séricas e índice de uremia según aclaramiento de creatinina medido.

\begin{tabular}{|c|c|c|c|c|c|c|}
\hline & \multicolumn{4}{|c|}{ Aclaramiento de creatinina $\left(\mathrm{ml} / \mathrm{min} / 1,73 \mathrm{~m}^{2} \mathrm{SC}\right)$} & \multirow[b]{2}{*}{$\mathbf{p}$} & \multirow[b]{2}{*}{$\begin{array}{c}\text { eta } \\
\text { cuadrado }\end{array}$} \\
\hline & $\begin{array}{c}>90 \\
(n=60)\end{array}$ & $\begin{array}{c}60-90 \\
(n=36)\end{array}$ & $\begin{array}{c}30-60 \\
(n=22)\end{array}$ & $\begin{array}{c}<30 \\
(n=26)\end{array}$ & & \\
\hline Creatinina sérica $(\mathrm{mg} / \mathrm{dl})$ & $0,69 \pm 0,15$ & $0,87 \pm 0,21$ & $1,20 \pm 0,57$ & $5,94 \pm 3,62$ & 0,00 & 0,624 \\
\hline Urea sérica $(\mathrm{mg} / \mathrm{dl})$ & $24,54 \pm 7,81$ & $28,24 \pm 10,14$ & $41,91 \pm 19,51$ & $129,63 \pm 60,07$ & 0,00 & 0,678 \\
\hline Índice de uremia & $1,92 \pm 0,47$ & $2,28 \pm 0,64$ & $3,30 \pm 1,44$ & $12,42 \pm 5,88$ & 0,00 & 0,705 \\
\hline
\end{tabular}

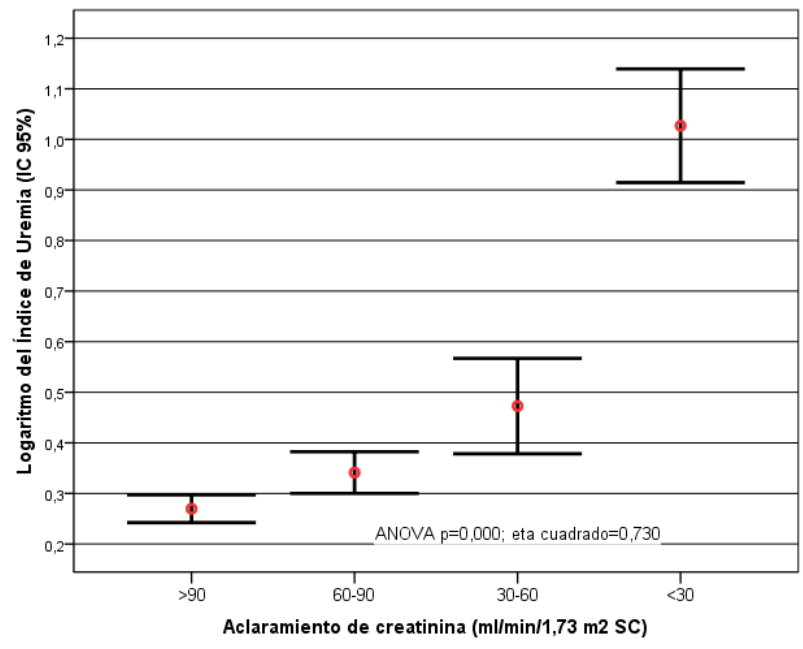

Gráfico 3. Logaritmo del índice de uremia y aclaramiento de creatinina según estadios de función renal.

Tabla 2. Índice de uremia, creatinina sérica y diversas fórmulas para estimar la tasa de filtración glomerular, según aclaramiento de creatinina medido.

\begin{tabular}{|c|c|c|c|c|c|c|}
\hline & \multicolumn{4}{|c|}{ Aclaramiento de creatinina $\left(\mathrm{ml} / \mathrm{min} / 1,73 \mathrm{~m}^{2} \mathrm{SC}\right)$} & \multirow[b]{2}{*}{$\mathbf{p}$} & \multirow[b]{2}{*}{$\begin{array}{c}\text { eta } \\
\text { cuadrado }\end{array}$} \\
\hline & $\begin{array}{c}>90 \\
(n=60)\end{array}$ & $\begin{array}{c}60-90 \\
(n=36)\end{array}$ & $\begin{array}{c}30-60 \\
(n=22)\end{array}$ & $\begin{array}{c}<30 \\
(n=26)\end{array}$ & & \\
\hline Índice de uremia & $1,92 \pm 0,5$ & $2,28 \pm 0,6$ & $3,30 \pm 1,4$ & $12,42 \pm 5,9$ & 0,00 & 0,705 \\
\hline Log Índice de uremia & $0,27 \pm 0,1$ & $0,34 \pm 0,1$ & $0,47 \pm 0,2$ & $1,03 \pm 0,3$ & 0,00 & 0,730 \\
\hline Creatinina sérica & $0,69 \pm 0,2$ & $0,87 \pm 0,2$ & $1,20 \pm 0,6$ & $5,9 \pm 3,6$ & 0,00 & 0,624 \\
\hline Fórmula peruana (Vásquez) & $111,2 \pm 24,3$ & $91,4 \pm 27,1$ & $70,0 \pm 34,7$ & $17,8 \pm 15,9$ & 0,00 & 0,640 \\
\hline Fórmula de Cockroft-Gault & $130,1 \pm 34,5$ & $100,8 \pm 37,4$ & $77,2 \pm 44,3$ & $18,0 \pm 18,1$ & 0,00 & 0,583 \\
\hline Fórmula de CKD-EPI & $110,9 \pm 16,6$ & $93,0 \pm 14,2$ & $85,5 \pm 22,7$ & $52,8 \pm 16,3$ & 0,00 & 0,604 \\
\hline
\end{tabular}


Tabla 3. Correlaciones con significancia estadística del índice de uremia y la creatinina sérica.

\begin{tabular}{lcccc}
\hline & \multicolumn{2}{c}{ Índice de uremia } & \multicolumn{2}{c}{ Creatinina sérica (mg/dl) } \\
\cline { 2 - 5 } & r de Pearson & $\mathbf{p}$ & $\mathbf{r}$ de Pearson & $\mathbf{p}$ \\
\hline Edad & 0,228 & 0,006 & - & $\mathrm{ns}$ \\
Índice de masa corporal & 0,219 & 0,008 & 0,238 & 0,004 \\
Agua corporal medida & $-0,185$ & 0,033 & - & $\mathrm{ns}$ \\
Sodio sérico & 0,33 & 0,000 & 0,247 & 0,006 \\
Potasio sérico & 0,407 & 0,000 & 0,256 & 0,004 \\
Fracción excretada de sodio & 0,222 & 0,021 & 0,313 & 0,001 \\
Fracción excretada de potasio & 0,485 & 0,000 & 0,496 & 0,000 \\
Ingesta diaria proteínas & 0,231 & 0,016 & 0,233 & 0,015 \\
\hline
\end{tabular}

Al comparar el valor predictivo del IU, la creatinina sérica y diversas fórmulas recomendadas en la literatura se encontraron asociaciones que se muestran en la tabla 2.

Al estudiar la correlación del IU y la creatinina sérica con otras variables, se encontró correlación entre el IU con otras variables importantes de la función renal sobresaliendo la del potasio y su fracción excretoria tubular (tabla 3). El sexo no mostró diferencias respecto al IU.

El IU promedio y su intervalo de confianza para personas con aclaramiento de creatinina mayor de 90 $\mathrm{ml} / \mathrm{min} / 1,73 \mathrm{~m}^{2}$ SC fue 1,95 (IC $\left.95 \% 1,93-2,08\right) \mathrm{mg} /$ $\mathrm{dl}$, para aclaramiento entre 60 y $90 \mathrm{ml} / \mathrm{min} / 1,73 \mathrm{~m}^{2} \mathrm{SC}$ fue 2,28 (IC 95\% 2,08-2,48) $\mathrm{mg} / \mathrm{dl}$, para aclaramiento entre 30 y $60 \mathrm{ml} / \mathrm{min} / 1,73 \mathrm{~m}^{2}$ SC fue 3,30 (IC $95 \%$ 2,71-3,89) $\mathrm{mg} / \mathrm{dl}$, y para aclaramiento menor de $30 \mathrm{ml} /$ min fue 12,48 (IC 95\% 10,15-14,69) mg/dl.

\section{DISCUSIÓN}

La tasa de filtración glomerular en personas sanas y con ERC puede ser estimada en la práctica clínica mediante el $\mathrm{ClCr}$, la cual tiene una correlación significativa $(\mathrm{r}=0,90)$ con el gold estándar (aclaramiento de inulina) ${ }^{(15,16,17)}$. Sin embargo, dada la magnitud variable de secreción tubular de creatinina y algunas dificultades en el proceso de recolección de orina que alteran la sensibilidad de la prueba, los estudios han planteado métodos alternativos que involucre un menor tiempo de recolección de la diuresis ${ }^{(17,18)}$, así como su estimación mediante fórmulas estandarizadas.

El IU es una expresión numérica que relaciona los niveles séricos de urea y creatinina, que en condiciones de homeostasis refleja el estado nutricional y la función renal de la persona sana. Si bien el presente estudio no consideró al aclaramiento de inulina que es el gold estándar, los resultados en términos generales evidencian relación estadísticamente significativa entre el IU y el $\mathrm{ClCr}$ tanto en personas sanas como en personas con diversos grados de ERC y diversidad de estructura corporal.

El paciente con ERC presenta una reingeniería en su composición corporal que lo hace vulnerable a la pérdida de la homeostasis, en este estudio el $26 \%$ de pacientes tuvieron algún grado de ERC y diferente estado de azoemia que traduce catabolismo proteico, en ese escenario, el IU y el Log IU mostraron un valor predictivo de la función renal superior a las fórmulas de estimación convencionalmente aceptadas, por lo que ambos marcadores pueden ser útiles en el diagnóstico precoz de la ERC.

La evaluación del estado nutricional es un factor importante en el control de los fenómenos de autorregulación corporal como el implicado en la homeostasis del medio interno y la respuesta inmunológica del huésped a una infección, en ese sentido, el análisis de biomarcadores como la urea y creatinina séricas de manera independiente reflejan la ingesta diaria proteica y la masa muscular activa respectivamente; sin embargo, su uso para estimar el $\mathrm{ClCr}$ se ve limitada por procesos de reabsorción $\mathrm{y}$ secreción tubular aumentadas respectivamente conforme progresa la ERC ${ }^{(4,5,19)}$. El presente estudio evidencia que el IU muestra también un valor predictivo del $\mathrm{ClCr}$ mayor a la creatinina y urea séricas.

La homeostasis del medio interno se refleja en el adecuado intercambio de sustancias gracias al potencial electrofisiológico de la membrana celular, 
misma que es regulado por la concentración sérica de potasio; el IU muestra correlación estadísticamente significativa con el potasio sérico y con la fracción excretoria del mismo, lo que traduce su importancia porque además muestra indirectamente el grado de compromiso de la función tubular.

La función renal que permite mantener el equilibrio hidroelectrolítico se refleja en el nivel de la natremia y su relación con el agua corporal total (ACT), este último presenta variaciones a expensas de los cambios en la masa magra y grasa corporal dependiente de la edad y el sexo ${ }^{(1,17)}$. El IU mostró correlación negativa significativa con el ACT, lo que traduce que niveles altos del IU se relaciona con disminución del ACT, hallazgo relevante, ya que se altera el volumen de distribución de múltiples sustancias involucradas en la regulación del medio interno. El IU tuvo también correlación significativa con el sodio sérico, que refleja la interacción entre la urea y el sodio séricos, ambos involucrados en la regulación del mecanismo de concentración y dilución urinarias ${ }^{(20)}$.

La estimación del $\mathrm{ClCr}$ a partir del uso de las fórmulas conocidas o de la creatinina sérica ha sido cuestionada por la literatura disponible en el contexto del paciente adulto mayor, quien debido a la disminución de su reserva fisiológica se hace vulnerable a la pérdida de la homeostasis corporal ${ }^{(21)}$, sin que ello signifique que la edad avanzada en una persona sana conlleve a ERC ${ }^{(1)}$. Nuestra población involucró $25,3 \%$ de personas adultas mayores de los cuáles el $30 \%$ fueron octogenarios; los resultados reflejan relación estadísticamente significativa del IU con la edad en comparación a la creatinina sérica, por lo que puede traducir de forma más confiable la función renal en este grupo etario.

Si bien el Log IU se presenta como el indicador que mejor predice la función renal en nuestra cohorte, se prefiere el IU por su aplicación práctica e interpretación inmediata al momento del abordaje del paciente con ERC.

En conclusión, el IU es un indicador del estado metabólico y nutricional que refleja la función renal medida por el $\mathrm{ClCr}$ con una precisión estadísticamente significativa en la persona sana y en el paciente con distintos grados de ERC. Además, traduce aspectos relevantes de la función tubular renal en comparación a la urea y creatinina séricas de forma independiente.
Esta herramienta sencilla permitirá un abordaje integral de forma práctica y podrá formar parte de marcadores útiles para amenguar la mortalidad en el grupo poblacional estudiado. Este estudio es coincidente con los trabajos originales que sugirieron el uso de este marcador, situación que para un país como el nuestro es de gran utilidad.

\section{Declaración de financiamiento y de conflictos de intereses:}

El estudio fue financiado por los autores. Declaramos no tener conflictos de intereses.

\section{Contribución de autoría:}

LECLL, JACZ: Participaron en el diseño del estudio, recolección de los datos, interpretación de los resultados y aprobación de la versión final a ser publicada.

\section{Correspondencia:}

Luis Enrique Cruz Llanos

Dirección: Av. Honorio Delgado 262. Urb. Ingeniería., San Martín de Porres,

Lima, Perú

Teléfono: 51948349208

Correo electrónico: luis.cruz.1@upch.pe

\section{REFERENCIAS BIBLIOGRÁFICAS}

1. Vilela A, Cieza J. Características de la estructura corporal, hábitos dietéticos y función renal a través de la vida, de personas saludables de clase media entre los 20 y 80 años de edad de Lima. Rev Med Hered. 2018; 29: 217-25.

2. Weiner ID, Mitch WE, Sands JM. Urea and Ammonia Metabolism and the Control of Renal Nitrogen Excretion. Clin J Am Soc Nephrol. 2015;10(8):144458.

3. García L, Santana S. Excreción urinaria de nitrógeno ureico estimada del índice urea-creatinina ajustado según el peso corporal. Rev Cubana Aliment Nutr. 2016; 26(1): 84-92.

4. Waikar S, Bonventre J. Can we rely on blood urea nitrogen as a biomarker to determine when to initiate dialysis? Clin J Am Soc Nephrol. 2016; 1: 903-4.

5. Santana S. Valores locales de referencia para la excreción urinaria de creatinina: Una actualización. Rev Cubana Aliment Nutr. 2014; 24 (2): 220-30.

6. Moller, E, McIntosh JF, Van Slyke DD. Studies of urea excretion II. J Clin Invest. 1928; 6(3): 427- 65. 
7. Losito A. Cardiovascular morbidity and longterm mortality associated with in hospital small increases of serum creatinine. J Nephrol. 2018;31(1):71-7.

8. Jabary N, Muñoz, M. Creatinina sérica y aclaramiento de creatinina para la valoración de función renal en hipertensos. Nefrología. 2006; 26(1): 64-73.

9. Thongprayoon C. Prognostic importance of low admission serum creatinine concentration for mortality in hospitalized patients. Am J Med. 2017; 130: 545-54.

10. Pinto de Souza S. Inverse association between serum creatinine and mortality in acute kidney injury. J Bras Nefrol. 2014; 36(4):469-75.

11. Tanaka S. Impact of blood urea nitrogen to creatinine ratio on mortality and morbidity in hemodialysis patients: The Q-Cohort Study. Sci Rep. 2017;7: 14901.

12. Josa-Laorden C. Valor pronóstico de la ratio urea / creatinina en la insuficiencia cardiaca descompensada y su relación con el daño renal agudo. Rev Clin Esp. 2018; 218(5):232-40

13. Lubowitz H, Slatopolsky E, Shankel S, Rieselbach RE, Bricker NS. Glomerular filtration rate. Determination in patients with chronic renal disease. JAMA.1967; 199:252-6.

14. Cieza JA, Casillas A, Da Fieno AM, Urtecho SB. Asociación del nivel de albúmina sérica y alteraciones de los electrolitos, gases sanguíneos y compuestos nitrogenados en pacientes adultos incidentes del servicio de emergencia de un hospital general. Rev Med Hered. 2016; 27:223-9.
15. Miller BF, Winkler AW. The renal excretion of endogenous creatinine in man. Comparison with exogenous creatinine and inulin. J Clin Invest. 1938; $17: 31-40$.

16. Milutinovic I, Cutler RE, Hoover P, Meijsen B, Scribner BH. Measurement of glomerular filtration rate in patients receiving repetitive hemodialysis. Kidney Int. 1975; 8:185-90.

17. Bjornsson TD. Use of Serum Creatinine Concentrations to Determine Renal Function. Clin Pharmacokinet. 1979; 4: 200-22.

18. Da Silva AB, Molina MCB, Rodrigues SL, Pimentel EB, Baldo MP, Mill JG. Correlation between the creatinine clearance in the urine collected during 24 hours and 12 hours. J Bras Nefrol. 2012; 45(9): 799805 .

19. Cockcroft DW, Gault MH. Prediction of creatinine clearance from serum creatinine. Nephron. 1976;16: 31-41.

20. Weiner ID, Mitch WE, Sands JM. Urea and Ammonia Metabolism and the Control of Renal Nitrogen Excretion. Clin J Am Soc Nephrol. 2015; 10(8):144458.

21. Rimon E, Kagansky N, Cojocaru L, Gindin J, Schattner A, Levy S. Can creatinine clearence be accurately predicted by formulae in octogenarian inpatients? Q J Med. 2004; 97:281 -7.

Recibido: $14 / 12 / 2020$

Aceptado: 23/09/2021 